\title{
Integrating Procedural Modelling Process and Immersive VR Environment for Architectural Design Education
}

\author{
Chun-Heng Lin ${ }^{1, a}$ and Pei-Hsien Hsu ${ }^{2}$ \\ ${ }^{1}$ NCTU, Architecture Department, 1001 University Road, Hsinchu, Taiwan, ROC \\ ${ }^{2}$ NCTU, Architecture Department, 1001 University Road, Hsinchu, Taiwan, ROC
}

\begin{abstract}
In the field of architecture, researches are being conducted for the issue of combining VR environment and procedural modelling. Instead of simply using VR as an visualization tool for procedural modelling, as in other researches, we argue that manipulations of $3 \mathrm{D}$ elements and adjustments of design parameters should be implemented in VR to provide further supports for a design process. An integrated system is thus proposed based on this augment, with a special focus on architectural design education. This paper presents the design of such a multiuser system, in which 3D modelling, procedural modelling and VR platform are integrated, aiming to support architectural design education.
\end{abstract}

\section{Motivation}

There has been a long history between architecture and virtual reality. As computer involves into design process, some of researches about digital media propose that virtual reality as medium for architecture design can allow students to experience almost actual perception from the immersive environment[1]. In addition, architecture navigation emerged in many experiments about VR (Virtual Reality)[2-4], they tried to make the scene look real and set up triggers in the scene to improve users' space perception. The device for VR display is changing with time, from computer screen to projection walls[5]. There is a big breakthrough of VR device compare to the old days. Based on the advancement of computing capability, VR equipment became more lightweight and affordable. The new VR device with powerful HMD (Head-mounted display) and natural human motions, brings back designers' imagination in virtual world. Therefore, it is a potential opportunity for architectural design education. The device allow students to access the immersive learning environment.

Procedural modelling is a great leap in CAAD (Computer-Aided Design and Drafting), and it drastically changes the way that designers think. Since 2007 to present, numerous experiments were conducted[6-7]. Procedural modelling has become an important architectural design methodology. With this methodology, designers can set the rules based on design concept to generate diverse form or space. Nevertheless, under the trend of procedural modelling, traditional architectural design education still use sketching and physical modelling as main methods to showcase the ideation. However, unlike experienced designers, students can only imagine or even guess the real scale of projects by current methods. According to the new VR breakthrough, there are some experiments about merging the new VR and procedural modelling process. Only real-time operation to objects, and

\footnotetext{
${ }^{\text {a } C o r r e s p o n d i n g ~ a u t h o r ~: ~ a j 71735 @ a r c h . n c t u . e d u . t w ~}$
} 
relevant parameters in VR environment are significant enough to provide a comprehensive understanding, in addition to navigation.

It is a new possibility for architecture students nowadays. They can learn in more instinctive and flexible environment with direct interaction to the scene, by the combination of immersive VR environment and procedural modelling process. This study suggests that VR platform is a more efficient tool for students to develop and discuss their design instead of a visualizing tool.

\section{Context}

In the previous paragraph, the relevant resource for our imagination is mentioned roughly, more details will be supplemented in the following contents.

\subsection{Medium in Architectural Design}

Medium contains design concept is employed by architects to share and discuss the ideas. A common way for them to express their design concept is $2 \mathrm{D}$ free-hand sketching. This method can be traced back to the time before Renaissance[8]. The ambiguity and amorphousness of sketching help architects to improve ideation process[9]. Sketching is also a simple method for brief discussion and exchange ideas[10]. The small-scale model is another medium. It helps to show design results in more precise way.

After computer got into design process, more and more studies are curious about the effects caused by digital media. Most of the studies think that digital involvement can enhance the efficiency of design process, reducing budget, expanding the complexity of form, and increasing experience[1112]. As designers become familiar with digital media, they tried to test the tool in diverse directions. At first, some of them applied the traditional design media, sketching and modelling, in digital way with their existed advantages in design[13-14]. On the other hand, some of them developed new design methodology. Create the forms and design results by setting a series of rules, which is procedural modelling. Furthermore, some of them considered about the experience of design process and presentation, so they focused on building an immersive world for design process.

\subsection{Procedural Modelling in Architecture Design}

In traditional CAAD modelling process, designers usually developed the form from concepts in mind first, then, they built it in CAAD tool piece by piece. Despite the concept of procedural modelling has been discussed over thirty years, it is not until about 2007, new digital tools help designers to think outside the box, and they break the linear design process[15]. That is to say, the way that designers develop form can be separated into many steps. Every step is a small function that can be reused in another design process. There are some essential advantages of procedural modelling such as data amplification capabilities, data compression, and unpredictability[16]. Designers can only design their own rules, and connect these rules with real-time data from sensors to design dynamic forms, instead of completing all form in advance. By the flexible procedural modelling tools, designers can also construct their own kit to design, and the kit can be easily share to the public.

\subsection{Spatial Perception and VR in Architectural Design Education}

It is still not common for architectural design education to use VR platform as presentation tool. Currently, students in class propose ideas and discuss by using sketches and models to imagine the project deliverables. In the early stage of architectural design, it depends on brief sketching to develop ideas. As the concept developed, it is time to prototype the abstract idea into space or buildings. In this stage, according to some researches, they think learning in the VR environment can improve novice's spatial cognition [17-18]. There are various studies about learning architectural design in VR[19-21]. 
VR technique development can be separate into several stages by the constrain of hardware[22]. At the beginning, the idea of immersive environment in real world is practiced by using heavy equipment and shows images on the screen. As projection and 3D image technique mature, CAVE (Cave Automatic Virtual Environment) is available with motion capture to give viewers surrounded by walls full interacting experience[23]. Because of the mobility, well-prepared platform, and the compatibility between display platform and other CADD tools, the new lightweight VR device shows more potential opportunities for architectural design education.

\subsection{Procedural Modelling in VR}

Combining procedural modelling and VR environment in real time is still a new issue. There are a few experiments between Grasshopper, a parametric modelling environment, and VR platform Unity 3D. In Gathmann's demo, he successfully transmits data from Grasshopper to Unity, and the program automatically create the collider of object in the scene[24]. The team, AWARE, tries to return data from Unity to Grasshopper to change the form by capturing the cursor position and object dimensions[25]. Although this case is done by capturing cursor position on 2D screen, the interaction is still a good example for getting operation states in VR platform. The experiment shared by Orange Jellies shows the parameters used in Grasshopper to user interface in Unity. In this case, users in VR platform can revise the value to change the form[26].

\section{System Overview}

As mentioned above, digital media involves in design process more and more often. Designers should also develop their original ideas at the same time, besides using digital media to represent conceptual ideas and to communicate with others. Based on the breakthrough of VR technique, this research expands on existing researches about digital media in design process. Proposing new design system which consists of existing 3D modelling platform and VR platform, and practicing it in architectural design education. It is not sufficient to learn architectural design only by navigation in VR environment, students can gain more feedback by operating and adjusting the form of design objects directly. The system also integrated the parametric operation in VR learning environment to visualize those rules behind forms. The visualization of design rules makes the discussion more understandable in class.

\subsection{System Workflow}

The system proposed by this study is shown as figure 1. 3D modelling platform and parametric modelling environment are setup on the server, and they are linked with VR platform through a specific protocol (TCP/IP), hence making distant connections over the internet feasible. The models in 3D modelling platform will be dynamically read by VR platform in the form of meshes. Some typical operations about objects in VR platform, such as translate, rotate, and scale, are sent back to 3D modelling platform to revise old models, then loading new models into VR platform again. If designers use parametric modelling environment as tool when modelling, they can choose the necessary parameters and show them on VR platform interface. Once the parameter is revised, the new value is sent back to parametric modelling environment, then new models are baked into 3D modelling platform automatically. Finally, those new models will be transmitted to VR platform once again. 


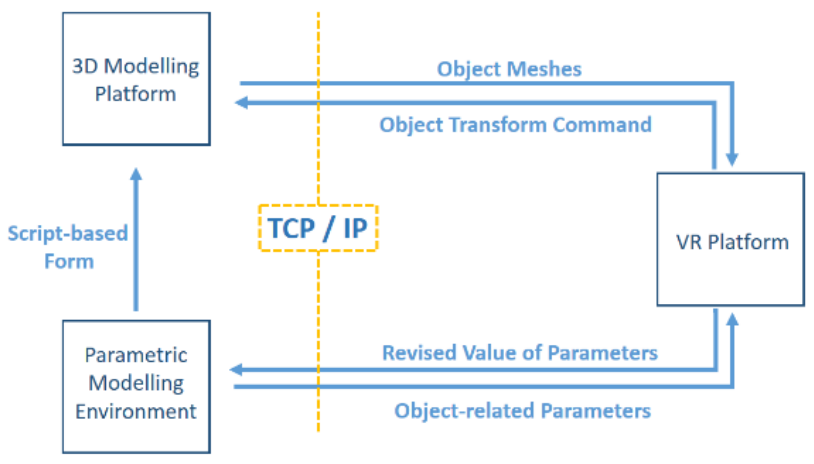

Figure 1. Data transmission framework

\subsection{Role of Designer and Participants in System}

There are two major roles in the system: designers and participants. To use the system, students in an architectural design class need to build their own model in 3D modelling platform and parametric modelling environment to present ideas and design concepts. Later upload their files to server. One of the design students in the class displays the model. If the model is built in parametric modelling environment, the student has to choose critical parameters for discussion or concept presentation in VR environment. Teachers and other students in the class, as the participants, can navigate the file with immersive environment. They can manipulate objects of the model at the same time, and the critical parameters chosen by designer are shown in the VR environment for value adjustment to present the concept, which is conducted by procedural modelling. The designers explain their design concepts in the VR environment, and the participants can understand the project not only by the explanation, but also can gain experience from their own perception. Moreover, they can revise the project to leave their advice or to discuss with designer. As a nature of virtual reality, the participants in this framework do not need to be in the same physical space. In addition, multiple version of revised model can be saved during the discussion. Students can also acess different version after the class, and save a new version to leave their revision. As shown in figure 2, with such a system, distance and asynchronous learning can thus be supported.

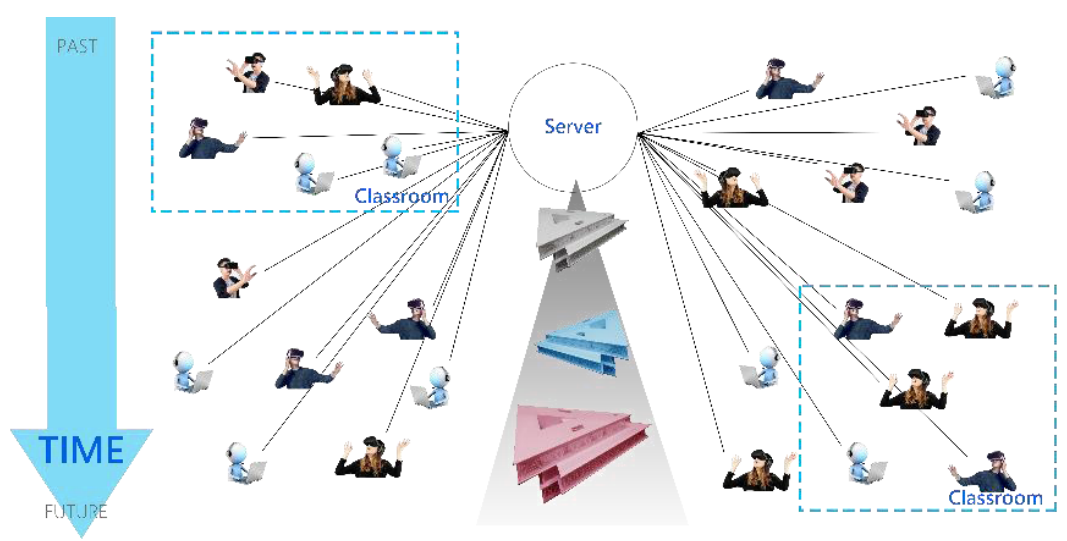

Figure 2. Architectural design education architecture 


\subsection{Education-Oriented Operation in VR World}

There are some operations need to be designed for the system proposed in this study. It is hard to do precise operations in the VR environment, yet the perception will become instinctive while it is free to control and interact with virtual objects without physical constrain. Therefore, as the scenario shown in figure 3, every part in the scene should be manipulated separately. For example, the walls interior can be moved or extended to test different spatial experience. A window can be put on many vertical surfaces to evaluate the best place for it in the project, and textures should be attached in simple way to get close to real perception of space, as well as the light. Those parameters, chosen by designer, involve in discussion in the VR environment as well. The precise value is not important at all, what is more is the relationship between variables and outcome that make the communication meaningful.

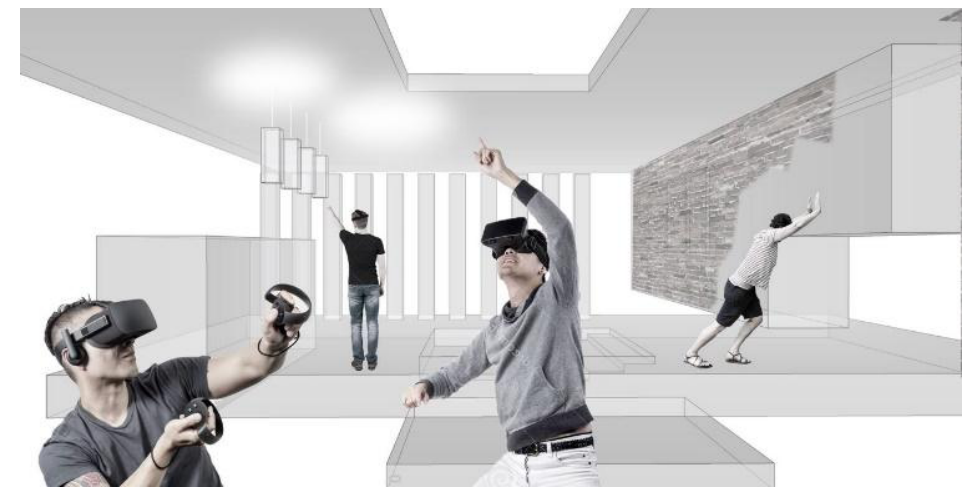

Figure 3. Scenario of architectural design education in the VR environment

\section{Conclusion}

Taking procedural modelling and new generation immersive VR as the basis, this paper presents a system framework, which integrates these two components, aiming to support architectural design education. Such a system framework is developed based on a design scenario: students and teachers in a design class simulate and discuss about an architectural design. By interacting with objects, in addition to the design parameters manipulation coming from the linked procedural modelling environment, "inside" an immersive VR environment. This leads to an integrated, multi-user system, which combines the strengths of procedural modelling along with $3 \mathrm{D}$ modelling environment and immersive virtual reality. Allowing designers to build 3D models that are automatically linked to an immersive VR environment to be accessed by all design class participants, in order to perform design simulations and discussions, which in turn, through the proposed system, feedback to the designers' 3D models and procedural model. As a work phase, the mechanism of data transmission, for both parametric data and geometric data between modelling and VR platforms, which is the key for the proposed system that have been developed and tested. Solutions to the possible conflicts between design parameters, structured in the procedural modelling platform, and direct object manipulations, supported inside VR environment, need to be developed. In addition to other tasks such as intuitive interfaces design for object manipulation and movement inside the VR environment, as the future steps toward the implementation of whole multi-user system framework for architectural design education.

\section{References}

1. S. Sven, et al. Educating architecture students to design buildings from the inside outExperiences from an research-based design studio. SSS9 Proceedings, Seoul (2013). 
2. M. Roupé, P. Bosch-Sijtsema, and M. Johansson. Interactive navigation interface for virtual reality using the human body. Comput Environ Urban Syst, 43, 42-50 (2014)

3. S. F. Kuliga, et al. Virtual reality as an empirical research tool-Exploring user experience in a real building and a corresponding virtual model. Comput Environ Urban Syst, 54, 363-375 (2015)

4. F. Castronovo, et al. An evaluation of immersive virtual reality systems for design reviews. Proceedings of the 13th CONVR, 22-29 (2013)

5. S. K. Ong, A. Y. C. Nee. Virtual and augmented reality applications in manufacturing. IFAC Proceedings, 46, 15-26 (2013)

6. S. Dritsas. Procedural Building Information Modeling for Digital Fabrication. CAADRIA 2015, 355-364 (2015)

7. D. Davis. Modelled on software engineering: Flexible parametric models in the practice of architecture. Diss. RMIT University (2013)

8. J. Whyte. Towards a new craft of architecture. BRI, 43, 2, 263-265 (2015)

9. S. Inoue, et al. Reductionism as a tool for creative exploration. Proceedings of 11 th EAD, Paris Descartes University, France, (2015).

10. G. Goldschmidt. Modeling the role of sketching in design idea generation. An Anthology of Theories and Models of Design. Springer London, 433-450, (2014)

11. D. Wu, et al. Cloud-based design and manufacturing: A new paradigm in digital manufacturing and design innovation. Comput Aided Des, 59, 1-14, (2015)

12. K. Vasylevska, I. Podkosova, H. Kaufmann. Walking in Virtual Reality: Flexible Spaces and Other Techniques. The Visual Language of Technique. Springer International Publishing, 81-97, (2015)

13. M. Evans, N. Aldoy. Digital Design Sketching using the Tablet PC. The Design Journal, 19, 5, 763-787 (2016)

14. R. Holden, J. Liversedge. Landscape architecture: an introduction. Laurence King Publishing, (2014)

15. P. Hsu Integration of Procedural Design and $3 D$ Virtual Environment to Support Collaboration for the Design of Built Environment. (2014)

16. R. M. Smelik, et al, A survey on procedural modelling for virtual worlds. CGF, 33, 6 (2014)

17. A. Angulo. On the design of architectural spatial experiences using immersive simulation. EAEA 11 Conference Proceedings, Envisioning Architecture: Design, Evaluation, Communication. Italy: Milan (2013)

18. Y. Chang. 3D-CAD effects on creative design performance of different spatial abilities students. JCAL, 30, 5, 397-407 (2014)

19. W. A. Abdelhameed. Virtual Reality Use in Architectural Design Studios: A case of studying structure and construction. Procedia Comput Sci, 25, 220-230 (2013)

20. D. Fonseca, et al. Visualization methods in architecture education using $3 D$ virtual models and augmented reality in mobile and social networks. Procedia Soc Behav Sci, 93, 1337-1343 (2013)

21. A. Brown, T. Green. Virtual reality: Low-cost tools and resources for the classroom. TechTrends, 60, 5, 517-519 (2016)

22. R. A. Earnshaw, M. A. Gigante, H. Jones. Virtual Reality Systems. Academic Press, (2014)

23. H. Creagh. Cave automatic virtual environment. Electrical Insulation Conference and Electrical Manufacturing \& Coil Winding Technology Conference, Proceedings. IEEE, (2003)

24. R. Gathmann. Grasshopper To Unity3D. Retrieved November 25, 2016, from https://vimeo.com/68066366

25. AWARE. Exchanging data between Unity and Grasshopper via UDP. Retrieved November 25, 2016, from http://www.aware-studio.com/exchanging-data-between-unity-and-grasshopper-via-udp/

26. O. Jellies. Send/Receive data between Grasshopper to Unity in realtime. Retrieved November 25 , 2016, from https://vimeo.com/153197500 